\title{
Community Care for Cancer Patients in Rural Areas: An Integrated Regional Cancer Center and Public Health Center Partnership Model
}

\author{
Jung Hun Kang, M.D., Chang Yoon Jung, M.D.*, Ki-Soo Park, M.D. ${ }^{\dagger}$, \\ Jung Sik Huh, M.D. ${ }^{\ddagger}$, Sung Yong Oh, M.D. ${ }^{\S}$ and Jung Hye Kwon, M.D." \\ Department of Internal Medicine, College of Medicine, Gyeongsang National University, Jinju, \\ *National Cancer Control Institute, National Cancer Center, Goyang, \\ ${ }^{\dagger}$ Department of Preventive Medicine, Institute of Health Sciences, College of Medicine, \\ Gyeongsang National University, Jinju, ${ }^{\ddagger}$ Department of Urology, Jeju National University, Jeju, \\ ${ }^{\S}$ Department of Internal Medicine, College of Medicine, Dong-A University, Busan, \\ "Department of Internal Medicine, College of Medicine, Chungnam National University, Daejeon, Korea
}

Purpose: The accessibility of medical facilities for cancer patients affects both their comfort and survival. Patients in rural areas have a higher socioeconomic burden and are more vulnerable to emergency situations than urban dwellers. This study examined the feasibility and effectiveness of a cancer care model integrating a regional cancer center (RCC) and public health center (PHC). Methods: This study analyzed the construction of a safety care network for cancer patients that integrated an RCC and PHC. Two public health institutions (an RCC in Gyeongnam and a PHC in Geochang County) collaborated on the development of the community care model. The study lasted 13 months beginning in February 2019 to February 2020. Results: The RCC developed the protocol for evaluating and measuring 27 cancer-related symptoms, conducted education for PHC nurses, and administered case counseling. The staff at the PHC registered, evaluated, and routinely monitored patients through home visits. A smartphone application and regular video conferences were incorporated to facilitate mutual communication. In total, 177 patients (mean age: 70.9 years; men: 59\%) were enrolled from February 2019 to February 2020. Patients' greatest unmet need was the presence of a nearby cancer treatment hospital (83\%). In total, $28(33 \%)$ and $44(52 \%)$ participants answered that the care model was very helpful or helpful, respectively. Conclusion: We confirmed that a combined RCC-PHC program for cancer patients in rural areas is feasible and can bring satisfaction to patients as a safety care network. This program could mitigate health inequalities caused by accessibility issues.

Key Words: Cancer care facilities, Home care service, Community networks, Telemedicine, Distance counseling, Rural health services
Received June 5, 2021

Revised September 21, 2021

Accepted September 22, 2021

Correspondence to

Jung Hye Kwon

ORCID:

https://orcid.org/0000-0002-5965-3204

E-mail: kwonjhye.onco@gmail.com

This study was supported by the National Cancer Center Grant (NCC1911270-1, NCC-1911277-1). 


\section{INTRODUCTION}

\section{General and specific background}

The accessibility of medical facilities affects both the comfort of cancer patients and their survival [1,2]. The availability of medical resources for receiving care in close proximity to one' s home is an important variable that influences the treatment of cancer patients, behavioral changes, and treatment compliance [3]. Therefore, accessibility is an important determinant of treatment outcomes as well as the financial condition of individual cancer patients, and insufficient accessibility is associated with cancer mortality in rural areas [4-6].

In South Korea (hereafter, Korea), 94\% of cancer patients, including $62 \%$ of patients who receive high-level treatment, are treated at general hospitals or other large facilities [7]. Since large-scale medical facilities tend to be concentrated in metropolitan areas, patients who live in rural areas experience a larger burden due to non-medical costs such as transportation and are more vulnerable to emergency situations than urban dwellers. This can lead to decreases in the quality of life and survival time among patients [8]. The continuum of care over the trajectory of a cancer case can be accomplished by providing coordinated care and home telehealth services, which in turn would contribute to improving patients' quality of life [9].

In Korea, public health centers (PHCs) and their subordinate bodies, public health units (PHUs), were established in 1962 in order to create a health care network and promote public health when the Health Center Act was revised and implemented. As of 2018, 254 public health centers and 1,338 public health units are in operation in Korea, covering all rural areas. In 2004, the Ministry of Health and Welfare established 12 regional cancer centers (RCCs) across each of the municipalities of Seoul to improve accessibility and reduce patients' socio-economic burden by increasing the concentration of health centers in the city.

\section{Debate over the issue}

Since 2018, the government has promoted a community care plan, which is a system that integrates and links the health and welfare systems, thereby enabling people with difficulties dur- ing independent daily life due to illness and aging to remain living in their home and receive care. The health management system must be strengthened through seamless collaboration between medical institutions and local governments in order to deliver effective community care [10]. Despite the principles for successful community care proposed by the Korean Medical Association and the pan-government policy drive [11], there is not yet a realistic domestic model targeted to cancer patients. In a previous study in which we attempted to examine the possibility of collaborative care service between RCCs and PHCs, the results revealed only the limitations to implementing such a model in Korea [12].

The Care Project was started in February 2019 by the National Cancer Center with the aim of improving care services for cancer patients in rural areas, who tend to be a relatively vulnerable population in terms of health outcomes. In particular, the Care Project primarily aimed to improve the quality of physical and mental care for cancer patients in rural areas and reduce their economic and social burden.

\section{Specific purpose of this study}

As part of the Care Project, an RCC and PHC collaborated on the basis of previous research to establish a care safety network, which could be referred to as a community care network, for rural cancer patients. The aim of this study was to examine the feasibility of a community cancer care model using a combined RCC-PHC and to report the provisional results of such a model.

\section{METHODS}

\section{Characteristics of organization and partnership}

In this study, a care model was designed for cancer patients on a collaborative basis by the Gyeongnam RCC in Jinju and a PHC in Geochang County. The Red Cross Hospital in Geochang County, which is affiliated with the Korean Red Cross, also participated in this project.

Both Jinju and Geochang County are located in the western part of the Korean province of Gyeongnam. The population of west Gyeongnam is 740,000 people, which comprises only $24 \%$ of the total population of Gyeongnam; however, it oc- 
cupies $57 \%$ of the total area of Gyeongnam, and its population centers are relatively more scattered than around Gyeongnam's larger cities. In addition, elderly people aged 70 or older comprise $33 \%$ of the population of west Gyeongnam, which is higher than the national average of $11 \%$ [13].

Founded in 2007, the Gyeongnam RCC conducts cancer treatment, hospice services, and survivorship programs for cancer patients who reside in west Gyeongnam. The PHC in Geochang County is a public institution with 11 PHUs and is responsible for administering various public health services such as home-based cancer care.

The cancer care services provided by RCCs and PHCs complement each other in terms of accessibility, the ability to link local welfare resources, and the presence of professional medical personnel and facilities. Two main entities, the Gyeongnam RCC and the PHC in Geochang County, formally agreed to collaborate on a project to improve the health outcomes of cancer patients at home beginning in January 2019 [14].

The program was conducted for patients who resided in Geochang County and were diagnosed with cancer according to their PHC registration information. A health care worker from the PHC visited each of the subjects to explain the purpose of the study and obtained informed consent.

\section{Unmet needs}

The unmet needs of enrolled patients were identified on an ad hoc basis and surveyed, and they included housekeeping help, transportation support, economic burden, psychological and physical strain, medical counseling and calls with staff, educational programs, reducing the number of hospital visits, and hopes for a nearby hospital offering cancer treatment. The subjects' unmet needs were measured using a 5-point Likert scale ranging from 0 (no need) to 4 (very high need).

\section{Measurement of the effect of the linked model}

To measure the severity of emergency situations for when referring patients to counseling by the Gyeongnam RCC, the Korean Triage and Acuity Scales (KTAS) was used, which records 5 levels of severity (level $1=$ resuscitation, level $2=$ emergency, level 3=urgent, level $4=$ less urgent, level $5=$ not urgent). Patient satisfaction was also assessed for patients who received linked services using a 5 -point Likert scale $(1=$ not at all helpful, 2=slightly helpful, 3=neutral, 4=moderately helpful, and $5=$ very helpful). The survey was administered over the telephone by a staff member at the Gyeongnam RCC 1 to 3 days after the consultation.

\section{Implementation of the care program}

Baseline data collection included demographic information such as socioeconomic status (household type, demographic characteristics, marital status, caregiver status, and medical insurance status), primary cancer, stage, main treatment hospital,

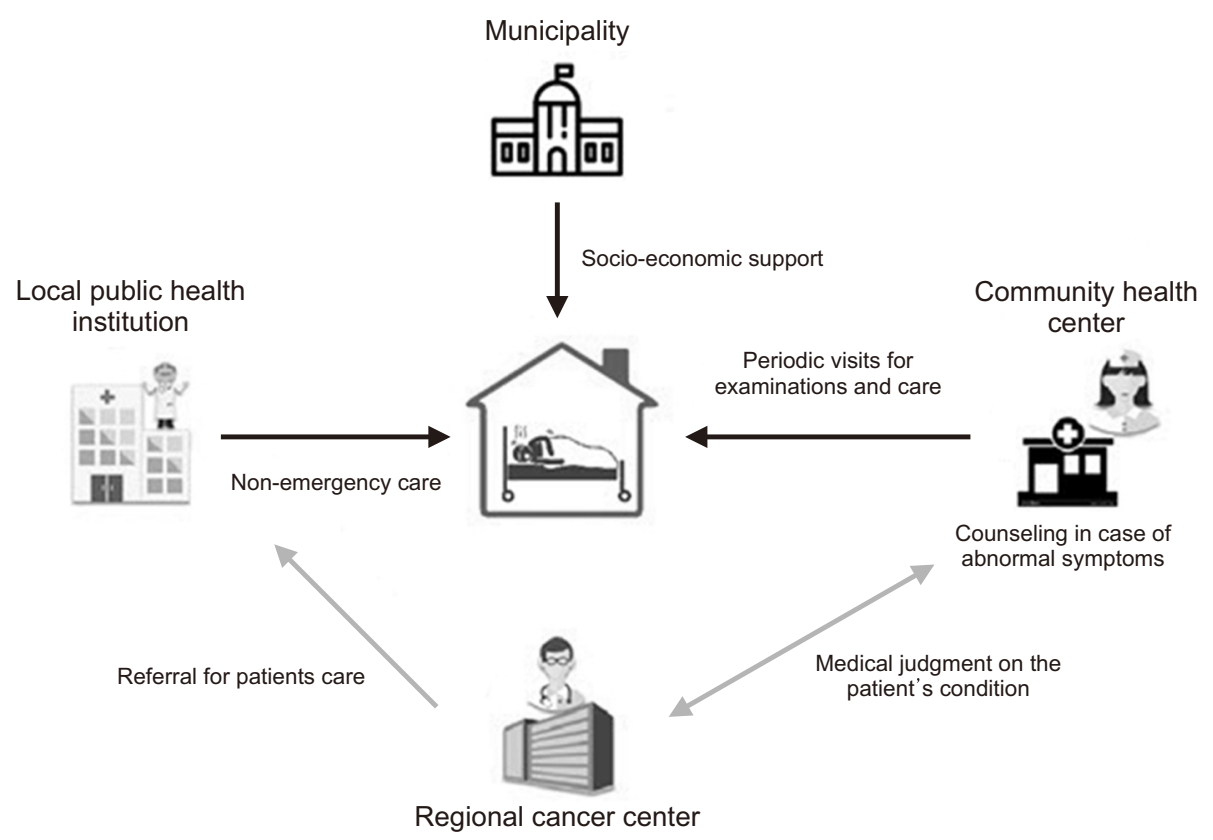

Figure 1. Schematic diagram of care system between regional cancer center and public health center. 
and other unmet needs. Patients who required welfare services such as housekeeping, bathing, or transportation assistance were linked to social workers at the county office to receive support.

The role of the PHC was to register cancer patients, conduct an initial evaluation, and routinely monitor patients during home visits, while the role of the Gyeongnam RCC was to develop a collaboration system, provide education on cancer care for nurses at PHCs, conduct case counseling, and make medical decisions when necessary. The Red Cross Hospital in Geochang County served as the facilitator, conducting laboratory tests and imaging workups and undertaking other supportive measures when needed by patients (Figure 1). When nurses at the PHC observed significant abnormalities in patients during home visits and requested a consultation by the Gyeongnam RCC, the medical oncologist at the Gyeongnam RCC made a medical decision on the issue and also coordinated the patient's referral as the team leader. The Gyeongnam RCC-PHC partnership model was revised and supplemented throughout based on regular feedback meetings with stakeholders from the PHC and Gyeongnam RCC.

\section{Ethical consideration and data analysis}

The partnership model was conducted prospectively as part of the Care Project, and the data were analyzed retrospectively. The study was reviewed and approved by the Institutional $\mathrm{Re}^{-}$ view Board of Gyeongsang National University Hospital College of Medicine (approval No. 2020-10-024-001).

Descriptive statistics were summarized as demographics and categorical variables of cancer characteristics.

\section{RESULTS}

\section{Implementation of the model}

The nurses from the PHC were required to visit patients' homes at least once per month for evaluations. However, more frequent visits were permitted depending on a patient's condition. A smartphone application was developed to facilitate patient consultations between the Gyeongnam RCC and the PHC (Figure 2). After obtaining informed consent from patients, nurses from the PHC entered information into the app

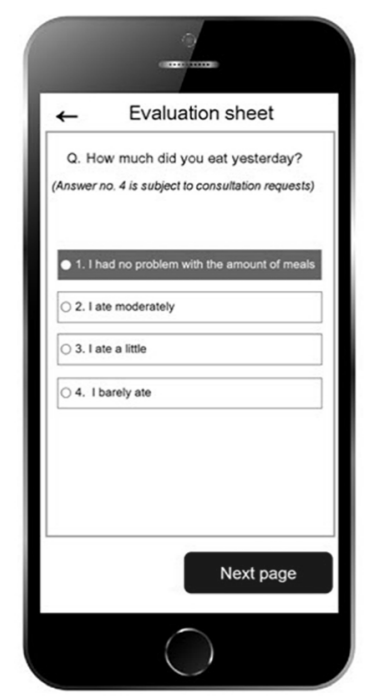

Figure 2. Screen shot of the cancer patient care service app.

after every visit on the condition of patients, including their vital signs and the severity of their symptoms. Symptoms included the patient's sense of well-being, constipation/diarrhea, frequency of meals, and sleep patterns, all of which were evaluated using a 4-point Likert scale (data not presented herein). An emergency message was automatically sent to the Gyeongnam RCC via the app to request a patient consultation when the patient had a body temperature of $\geq 38.0^{\circ} \mathrm{C}$, had a pulse rate of $\geq 100$ beats/minute, had a systolic blood pressure of $\leq 90 \mathrm{mmHg}$, or were given a 4 out of 4 rating on the Likert scale for his or her sense of well-being, constipation/diarrhea, frequency of meals, or amount of sleep. The coordinator at the Gyeongnam RCC made a call to visiting nurses from the PHC to confirm that counseling was needed and then connected them with the medical oncologist at the Gyeongnam RCC for advice.

In addition to the emergency system, recurring video meetings (called online rounds) were conducted every 2 weeks between health care professionals from the PHC and the medical oncologist from the Gyeongnam RCC to discuss individual patients' cases. Based on the discussion of the case, the medical oncologist from the Gyeongnam RCC sometimes examined the patient's condition by conducting a home visit. Parallel to the video conference, the Gyeongnam RCC conducted an education program for health care workers from the PHC about control of cancer symptoms and emergency care. We also developed counseling protocols for nurses at the PHC about 27 
common cancer-related symptoms (Supplement 1).

As the first task of the consultation protocol, a list of common symptoms eligible for counseling and other related materials were created independently by 3 medical oncologists (K.J.H, G.S.I, L.G.W.). The contents were revised until a consensus was reached between the doctors. Communication between the physicians at the Gyeongnam RCC and the Red Cross Hospital was facilitated via phone calls and chatting.

\section{Patient characteristics}

A total of 177 patients were enrolled from February 2019 to February 2020 (Table 1). In total, 84\% of patients were over 60 years old, including $21 \%$ of subjects who were over 80 years old. Furthermore, $12 \%$ of patients received Medical Aid, while $87 \%$ received coverage from National Health Insurance. In addition, $24 \%$ of the subjects lived in single-person households. The most common cancer was gastrointestinal cancer (33\%), followed by hepatobiliary tumors (15\%) and lung cancer (15\%). Moreover, $27 \%$ of patients were unaware of their cancer stage. Most patients were diagnosed within the previous 3 years, and most received active cancer treatment at hospitals concentrated in Daegu and Seoul (Table 2).

Table 1. Demographics ( $N=177)$.

\begin{tabular}{lc}
\hline \multicolumn{1}{c}{ Factors } & $\mathrm{n}(\%)$ \\
\hline Sex & $104(59)$ \\
Male & $73(41)$ \\
Female & \\
Age & $29(16)$ \\
$\geq 30$ to $<60$ & $44(25)$ \\
$\geq 60$ & $67(38)$ \\
$\geq 70$ & $37(21)$ \\
$\geq 80$ & \\
Health insurance status & $13(7)$ \\
Medical aidrecipient & $9(5)$ \\
Second-lowest income bracket & $153(87)$ \\
National Health Insurance & $2(1)$ \\
Unknown & \\
Marital status & $134(76)$ \\
Married & $40(22)$ \\
Unmarried & $3(2)$ \\
Unknown & \\
Caregiver status & \\
None & $110(62)$ \\
Spouse & $24(14)$ \\
Other family member & \\
& \\
\hline & \\
\hline & \\
&
\end{tabular}

\section{Symptoms caused by cancer and unmet needs}

A total of 141 out of 177 patients complained of cancer- or treatment-related symptoms following their cancer diagnosis, with 54 patients citing one symptom, 46 citing two symptoms, and 39 citing three symptoms. Frequent physical symptoms included fatigue (70\%), insomnia (51\%), pain (31\%), and depression (30\%). In total, $76 \%$ of the patients lived with family members, and $90 \%$ responded that they had someone to take care of them when they were sick.

The most common unmet need among the patients was a nearby cancer treatment hospital. Of the 177 patients enrolled, $95(54 \%)$ answered that they hoped highly for a nearby cancer

Table 2. Cancer-related Characteristics ( $N=177)$.

\begin{tabular}{|c|c|}
\hline Category & $\mathrm{n}(\%)$ \\
\hline \multicolumn{2}{|l|}{ Primary cancer } \\
\hline Gastrointestinal & $58(33)$ \\
\hline Hepatobiliary & $27(15)$ \\
\hline Lung & $26(15)$ \\
\hline Genitourinary and gynecologic & $22(12)$ \\
\hline Breast & $20(11)$ \\
\hline Head and neck & $9(5)$ \\
\hline Others & $15(8)$ \\
\hline \multicolumn{2}{|l|}{ Stage } \\
\hline । & $48(27)$ \\
\hline$\|$ & $27(15)$ \\
\hline$\| \mid$ & $35(20)$ \\
\hline IV & $19(11)$ \\
\hline Unknown & $48(27)$ \\
\hline \multicolumn{2}{|l|}{ Duration of disease (yr) } \\
\hline$<1$ & $65(37)$ \\
\hline $1 \sim 2$ & $20(11)$ \\
\hline $2 \sim 3$ & $42(24)$ \\
\hline$\geq 3$ & $28(16)$ \\
\hline Unknown & $21(10)$ \\
\hline \multicolumn{2}{|l|}{ Distance to the main treatment hospital } \\
\hline City 1 hour away (Daegu) & $77(44)$ \\
\hline City 1 hour away (Seoul) & $72(41)$ \\
\hline City 2 hours away (Busan) & $15(8)$ \\
\hline City 1 hour away (Jinju) & $8(5)$ \\
\hline Others & $5(3)$ \\
\hline \multicolumn{2}{|l|}{ Cancer treatment } \\
\hline Operationalone & $70(39)$ \\
\hline Chemotherapy and operation & $26(15)$ \\
\hline Chemotherapy alone & $25(14)$ \\
\hline Chemotherapy, radiotherapy operation & $19(11)$ \\
\hline Radiotherapy alone & $8(5)$ \\
\hline Others & $29(16)$ \\
\hline
\end{tabular}


treatment hospital, and 51 (29\%) said they hoped very highly. The next most common unmet need was a decrease in the number of required visits to treatment hospitals. A total of 130 patients $(73 \%)$ highly or very highly hoped for fewer hospital visits. The next most common unmet needs were education related to cancer and counseling calls (Figure 3 ).

\section{Evaluation of the partnership model}

The medical oncologist from the Gyeongnam RCC conducted 51 home visits (29\% of the 177 patients) to further assess patients' condition.

The PHC submitted a total of 85 counseling requests. Of them, 44 cases $(52 \%)$ received a KTAS rating of 5, 30 cases received a KTAS rating of $4(35 \%), 9$ received a KTAS rating of $3(11 \%)$, and 2 received a KTAS rating of $2(2 \%)$.

Hospital visits were recommended for 35 patients (41\%), of whom 26 (74\%) went to the Red Cross Hospital in Geochang County (the nearby public hospital) and 9 (26\%) went to the Gyeongnam RCC. In the service satisfaction survey, 44 subjects
$(52 \%)$ answered that the program was moderately helpful, and $28(33 \%)$ answered that it was very helpful, indicating that the majority of patients were satisfied with the Gyeongnam RCCPHC partnership program. Only 4 (5\%) and 9 (10\%) subjects, respectively, responded that the program was slightly helpful or not at all helpful.

\section{Representative cases}

Two representative cases that demonstrate the purpose of the partnership model are discussed in this section (Figure 4). Sudden short-term memory loss and difficulty with speech were observed in an 81-year-old female patient with a history of hematologic malignancy during a routine home visit by a nurse from the PHC. She lived alone, and the nurse requested counseling without citing any particular disease since the symptoms were minor. It was then urgently recommended that the patient be transferred to a large hospital for workup and treatment with a suspected cerebral stroke or brain metastasis. She was diagnosed with cerebral infarction and received treat-
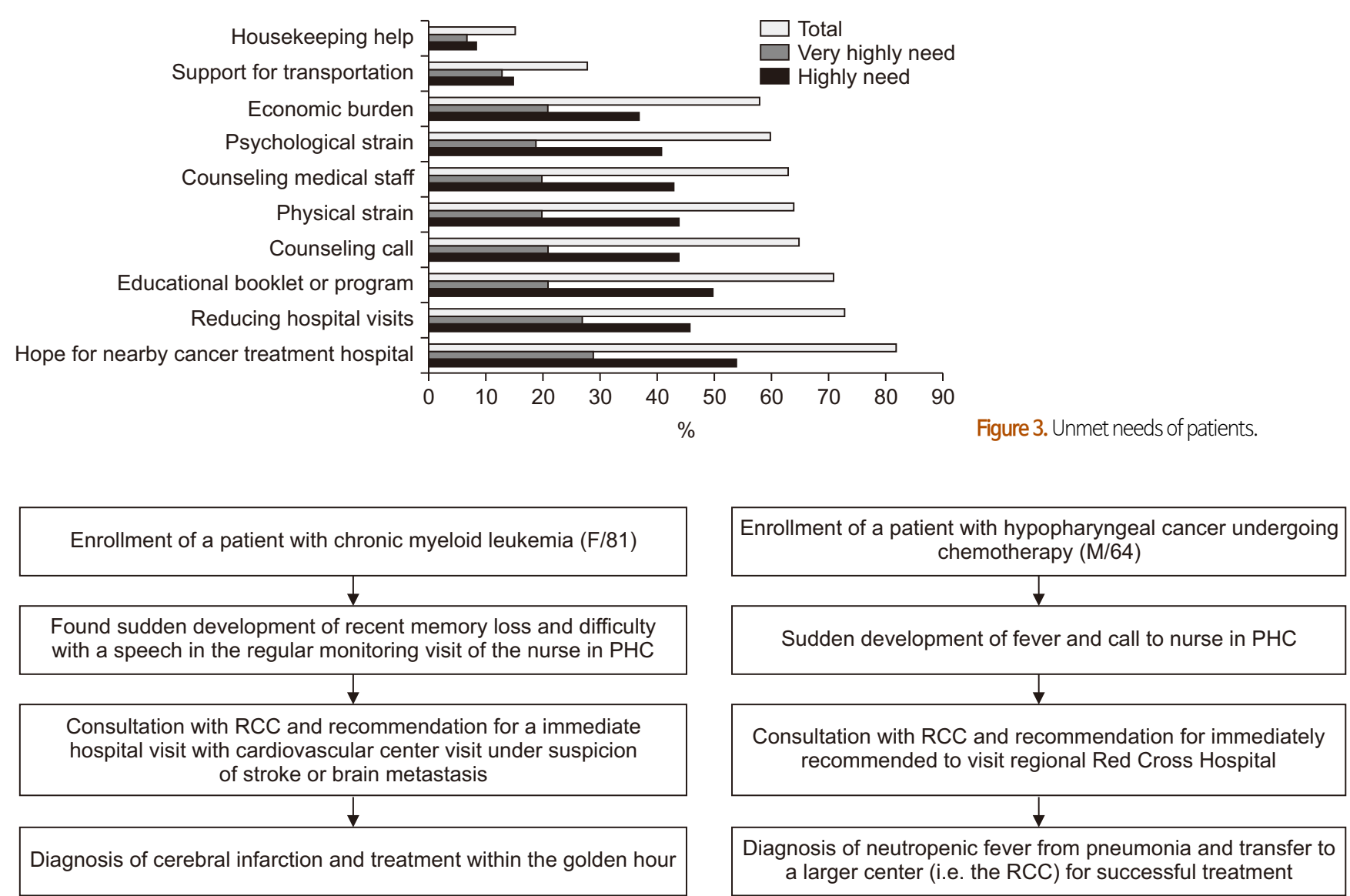

Figure 4. Summary of representative cases demonstrating collaborative care service. PHC: public health center, RCC: regional cancer center. 
Table 3. Cancer Community Care Model.

\begin{tabular}{|c|c|c|c|c|c|}
\hline $\begin{array}{l}\text { First author } \\
\text { [ref no.] }\end{array}$ & Subject & Country & Setting & Flow of cooperative service & $\begin{array}{l}\text { Stage of model } \\
\text { implementation }\end{array}$ \\
\hline Kim [16] & Terminal cancer & South Korea & Urban & Community care center $\leftrightarrow$ public health center & Execution stage \\
\hline Hojjat-Assari [17] & Terminal cancer & Iran & Urban & Comprehensive health center $\leftrightarrow$ health base $\leftrightarrow$ home care centers & Development stage \\
\hline Bull [18] & Life-limiting illness & United states & Urban and rural & Hospital $\leftrightarrow$ care facility $\leftrightarrow$ clinic & Execution stage \\
\hline Russell [19] & Cancer survivor & Australia & Urban and rural & Hospital-based oncology service $\rightarrow$ community-based health service & Development stage \\
\hline Ourstudy & All stages of cancer & Korea & Rural & Tertiary cancer center $\leftrightarrow$ public health center & Execution stage \\
\hline
\end{tabular}

ment within the golden hour. In another case, a 64-year-old patient undergoing chemotherapy for hypopharyngeal cancer was observed. A consultation was requested after he suddenly developed a fever. The patient's body temperature was $38.3^{\circ} \mathrm{C}$, and his pulse rate was 110 beats per minute. A visit to the Red Cross Hospital was immediately recommended for workup. The doctor at the hospital was given a brief explanation of the situation before the patient's visit so that the patient could undergo the appropriate corresponding examination. He was diagnosed with right lower lobe pneumonia with an absolute neutrophil count of 240 cells/ $\mu \mathrm{L}$ and oxygen saturation of $89 \%$. He was transferred to the emergency room at the Gyeongnam RCC. He fully recovered after 2 weeks of taking antibiotics and receiving other supportive measures. These cases demonstrate the usefulness and practicability of an RCC-PHC partnership model for socioeconomically vulnerable cancer patients in rural areas. There were many other subjects whose symptoms were treated at the nearby Red Cross Hospital.

\section{DISCUSSION}

Community care is an important global trend in today's rapidly aging society. In order to achieve effective community care, a variety of models should be developed which consider patients' illnesses, residential environments, and available support resources in their communities. The aim of this study was to establish the first practical community care model in which an RCC and PHC collaborated to provide treatment for cancer patients living in rural areas. This model has the advantage of supporting patients during the entire trajectory of their cancer treatment by implementing an RCC-PHC safety network to help with the early detection of medical problems and resolve minor problems using regional medical resources.
Kim et al. examined a community-based palliative care model for advanced cancer patients living in urban areas [15]. The model was a nursing care- and PHC-based palliative care program. The model first established a palliative care center for terminal cancer patients under the leadership of a PHC in the city, and care services were then provided by nurses during home visits. Although the program's use of community health care resources to provide support to patients is similar to our program, there are several essential differences between the models. First, our network program included an RCC, a local Red Cross Hospital, and a PHC, while the model in the study by Kim et al. included a PHC and a nursing college. The networking consultation program in our study was designed so that each entity shared a role and organically complemented the other entities in order to provide timely medical services to cancer patients.

Second, while patients were limited to urban-dwelling terminal cancer patients in the model by Kim et al., all ruraldwelling cancer patients were eligible for participation in our model. Lastly, we incorporated information and communication technology in our model including online tools and an application for symptom screening to facilitate communication between health care personnel. Such tools were successfully incorporated into our model.

Community care models for cancer patients have also been attempted in other countries (Table 3). However, those studies differ from one another with regard to their collaboration partners and target subjects depending on the various medical systems and available resources of each country.

Our study showed that a linked RCC-PHC model could enhance the social safety net in terms of addressing the emergency situations of cancer patients who live in rural areas where there tend to be relatively insufficient medical resources. Our study is meaningful in that it outlined the first practical 
model for community care of cancer patients in rural areas, which is currently a high-priority national policy task. It is possible in the future to expand the scope of the program to make it an additional collaborative safety network system for other chronic illnesses including cardiovascular disease and heart failure in rural areas. Regional centers for serious illnesses including respiratory centers, cardiovascular centers, and joint centers have been established in Korea. Expansion of our model to those centers could help mitigate health inequalities caused by accessibility issues.

Our model has another advantage since it not only improves cancer patient care but also can be expanded to include a cancer survivorship program. As of now, there are 12 cancer survivorship centers in Korea, but all of them are located in large cities. Accessibility is essential, however, given the nature of the survivorship program, and many cancer patients who live in rural areas are currently unable to take advantage of such services. Based on trust and our experiences with mutual cooperation thus far, we are attempting to implement cancer survivorship programs at regional cancer centers in rural areas through the dynamic division of roles [16].

Although the government has deployed nurses in each township area for community care, their unique role as medical personnel is still uncertain. If these nurses are involved in an RCC-PHC partnership model and play a role in caring for cancer patients, the quality of community care could be improved. Another advantage of our model is that it does not require additional investment in social resources for community care since it simply requires cooperation between existing medical personnel and systems rather than new investment.

Despite these several advantages, this network model still has some limitations. First, the government welfare system does not always have enough resources available to care for all patients with cancer. Despite the necessity of social welfare support for patients to be able to receive treatment at home, the current level of resources is far from enough. For example, even though many patients require transportation assistance to travel to and from hospitals, transportation assistance is rarely available. Since the national social welfare system is based on income, patients who are not eligible for Medical Aid cannot receive such social welfare services. Second, there is a shortage of medical personnel who can participate in this project. To implement this community care model, PHCs need to change their goals from existing preventive and health promotion $\mathrm{ac}^{-}$ tivities to caring for patients with serious illnesses. In order to achieve this shift in the role of PHCs, efforts to adjust incentives and work assessment areas should be made at the national or central department level, such as within the Ministry of Health and Welfare, rather than the local government level, such as city or county governments. The third limitation is that limited access to medical information makes it difficult for careful decision-making when it comes to patients' medical needs. Since such a program depends on the medical history of patients to understand their condition, the risk of misjudgment exists without first-hand knowledge of patients' conditions. The final limitation is that, at the time of this study, there were no existing tools to measure the effectiveness of the model. To overcome this, we are in the process of conducting qualitative research on patients' experiences with the program's services.

In conclusion, we demonstrated the feasibility of an RCCPHC partnership model to achieve community care for cancer patients who live in rural areas without requiring additional resource investment. However, in order to implement this model nationwide, efforts should be made at the national level to eliminate various obstacles.

\section{ACKNOWLEDGMENTS}

The authors greatly appreciate officials from the Geochang Public Health Center, especially nurse Yu Jin Lim and Dr. Jun Choi from the Geochang Red Cross Hospital for their active participation in the project.

\section{CONFLICT OF INTEREST}

No potential conflict of interest relevant to this article was reported.

\section{ORCID}

Jung Hun Kang, https://orcid.org/0000-0001-5013-2683

Chang Yoon Jung, https://orcid.org/0000-0001-7750-4213

Ki-Soo Park, https://orcid.org/0000-0001-5571-3639

Jung Sik Huh, https://orcid.org/0000-0003-2767-4390 
Sung Yong Oh, https://orcid.org/0000-0002-8321-4113

Jung Hye Kwon, https://orcid.org/0000-0002-5965-3204 article: all authors. Final approval of the version to be published: all authors.

\section{AUTHOR'S CONTRIBUTIONS}

Conception or design of the work: JHK, CYJ, JHK. Data collection: all authors. Data analysis and interpretation: JHK, JHK. Drafting the article: all authors. Critical revision of the

\section{SUPPLEMENTARY MATERIALS}

Supplementary materials can be found via https://doi. org/10.14475/jhpc.2021.24.4.226.

\section{REFERENCES}

1. Belasco EJ, Gong G, Pence B, Wilkes E. The impact of rural health care accessibility on cancer-related behaviors and outcomes. Appl Health Econ Health Policy 2014;12:461-70.

2. Aboagye JK, Kaiser HE, Hayanga AJ. Rural-urban differences in access to specialist providers of colorectal cancer care in the United States: a physician workforce issue. JAMA Surg 2014;149:537-43.

3. Kreling BA, Canar J, Catipon E, Goodman M, Pallesen N, Pomeroy J, et al. Latin American Cancer Research Coalition. Community primary care/academic partnership model for cancer control. Cancer 2006;107 (8 Suppl):2015-22.

4. Kim NY, Oh JS, Choi Y, Shin J, Park EC. Relationship between socioeconomic status and accessibility for endoscopic resection among gastric cancer patients: using National Health Insurance Cohort in Korea: poverty and endoscopic resection. Gastric Cancer 2017;20:61-9.

5. Adams SA, Choi SK, Khang L, A Campbell D, Friedman DB, Eberth JM, et al. Decreased cancer mortality-to-incidence ratios with increased accessibility of federally qualified health centers. J Community Health 2015;40:633-41.

6. Ramsey SD, Bansal A, Fedorenko CR, Blough DK, Overstreet KA, Shankaran V, et al. Financial insolvency as a risk factor for early mortality among patients with cancer. J Clin Oncol 2016;34:980-6.

7. Youn K. Comparisons of health care utilization patterns and outcome for National Health Insurance and Medical Aid Program cancer patients. J Health Info Stat 2014;39:42-59.

8. King M, Jones L, Richardson A, Murad S, Irving A, Aslett H, et al. The relationship between patients' experiences of continuity of cancer care and health outcomes: a mixed methods study. Br J Cancer 2008;98:529-36.

9. Gorin SS, Haggstrom D, Han PKJ, Fairfield KM, Krebs P, Clauser SB. Cancer care coordination: a systematic review and meta-analysis of over 30 years of empirical studies. Ann Behav Med 2017;51:532-46.

10. Braun K, Conybeare C. Active aging in Asia: community care policies and programs in Hong Kong, Japan, Korea, and Singapore. Innov Aging 2017;1 (suppl 1):685.

11. Kang TK. Principles of community integrated care (community care). Healthcare Policy Forum 2019;17: 6-10.

12. Song H-N, Kang MH, Lee GW, Kim HG, Lee WS, Kang JH, et al. Assessment of the effect of a public health clinics' home-based terminal cancer patient management in collaboration with a regional cancer center. Korean J Hosp Palliat Care 2013;16:10-9.

13. gyeongnam.go.kr [Internet]. Changwon: Gyeongnam Province: 2021 [cited 2021 Mar 11]. Available from: http://www.gyeongnam.go.kr/ index.gyeong? menuCd=DOM_000000106004010000.

14. Geochang county: vision and promise of Geochang county governor [Internet]. Geochang: Geochang County; 2021 [cited 2021 Mar 15]. Available from: https://www.geochang.go.kr/mayor/data/pdf/2019/3-8.pdf.

15. Kim SN, Choi SO, Shin SH, Ryu JS, Baik JW. Development of a community-based palliative care model for advance cancer patients in public health centers in Busan, Korea. Cancer Res Treat 2017;49:559-68.

16. Geochang county: enthusiastic response to survivorship program for cancer patients and families [Internet] Geochang: Geochang County; 2021 [updated 2019; cited 2021 Mar 23]. Available from: http://mediaissue.net/View.aspx? No=294580.

17. Hojjat-Assari S, Rassouli M, Madani M, Heydari H. Developing an integrated model of community-based palliative care into the primary health care (PHC) for terminally ill cancer patients in Iran. BMC Palliat Care 2021;20:100.

18. Bull J, Kamal AH, Harker M, Taylor DH Jr, Bonsignore L, Morris J, et al. Standardization and scaling of a community-based palliative care model. J Palliat Med 2017;20:1237-43.

19. Russell L, McIntosh R, Martin C, Scott J, Soo WK, Zappa B, et al. A model of cancer survivorship care within a community health setting: the Good Life Cancer Survivorship program. J Cancer Surviv 2020;14:36-42. 